\title{
On the approximate analytical solution to non-linear oscillation systems
}

\author{
Mahmoud Bayat* and Iman Pakar \\ Department of Civil Engineering, Mashhad Branch, Islamic Azad University, Mashhad, Iran
}

Received 22 August 2011

Revised 14 January 2012

\begin{abstract}
This study describes an analytical method to study two well-known systems of nonlinear oscillators. One of these systems deals with the strongly nonlinear vibrations of an elastically restrained beam with a lumped mass. The other is a Duffing equation with constant coefficients. A new implementation of the Variational Approach (VA) is presented to obtain highly accurate analytical solutions to free vibration of conservative oscillators with inertia and static type cubic nonlinearities. In the end, numerical comparisons are conducted between the results obtained by the Variational Approach and numerical solution using Runge-Kutta's [RK] algorithm to illustrate the effectiveness and convenience of the proposed methods.
\end{abstract}

Keywords: Variational Approach (VA), nonlinear vibrations, analytical methods, Runge-Kutta's [RK] algorithm

\section{Introduction}

In engineering and physics, differential equations that govern dynamic systems have widely been investigated recently $[1,2]$. Nonlinear dynamic problems have fascinated applied mathematicians, physicists and engineers for a long time. Over the past few decades applications in solid and structural mechanics as well as fluid mechanics have appeared, and there is now a widespread interest in the engineering and applied science communities in nonlinear oscillators, strange attractors, chaotic and dynamical systems theories. In spite of the great elegance and simplicity of such equations, the solutions of specific problems are significantly cumbersome to derive [3]. Finding innovative methods to analyze and solve these equations has been an interesting subject in the field of ordinary and partial differential equations and dynamical systems [4-6]. For most real-life nonlinear problems, it is not always possible and sometimes not even advantageous to express exact solutions of nonlinear differential equations explicitly in terms of elementary functions or independent spatial and/or temporal variables; however, it is possible to find approximate solutions. In recent years, many ingenious analytical methods have been developed for solving different kinds of strongly nonlinear equations, such as homotopy perturbation method [7,8], energy balance method [9-12], variational iteration method [13,14], variational approach [15-18], iteration perturbation method [19], Hamiltonian Approach [20], max-min approach [21-24], parameter expansion method [25], and other analytical and numerical methods [26-30].

The Variational Approach (VA) has recently become an efficient analytical technique in solving nonlinear problems. In this method, the first iteration leads to a highly accurate solution as compared with other analytical methods such as homotopy perturbation method, parameter expansion method, homotopy analysis method. Thus, an attempt has been made to apply VA for solving the governing equation of an elastically restrained beam with a lumped mass and a Duffing equation with constant coefficients. The investigations of cantilever beam models have been studied by

*Corresponding author: Mahmoud Bayat, Department of Civil Engineering, Mashhad Branch, Islamic Azad University, Mashhad, Iran. E-mail: mbayat14@yahoo.com. 
many researchers. Hamdan and Shabahen [31] studied the large amplitude free vibrations of a uniform cantilever beam carrying an intermediate lumped mass and rotary inertia. Hu et al. [32] developed the work on the nonlinear vibration of a cantilever with a Derjaguin-Müller-Toporov contact end by considering the principal resonance. Ke et al. [33] analyzed the flexural vibration of a functionally graded cantilever beam. Chen and Chen [34] applied the differential transformation method to cope with a fifth-order nonlinear problem. Mehdipour et al. [35] used the energy balance method for solving the nonlinear equation of cantilever beams. In the present work, the prime objective is to explore the application of the VA method for the fifth-order strongly nonlinear problem. The paper has been collocated as follows: primarily, the basic concept of He's variational approach is described. This is followed by the basics of the Ruge-Kutta's algorithm. Then the mathematical formulations of the problems are considered. In the next section, some applications of He's variational approach have been studied, to demonstrate the applicability and preciseness of the method. Some comparisons between analytical and numerical solutions are presented and eventually, it is shown that VA can converge to a precise cyclic solution for nonlinear systems.

\section{Basic concept of Variational Approach (VA)}

He suggested a variational approach which is different from the known variational methods in open literature [16]. Hereby a brief introduction of the method is presented. Consider the following differential equation.

$$
\ddot{u}+f(u)=0
$$

Its variational principle can be easily established utilizing the semi-inverse method [16];

$$
J(u)=\int_{0}^{T / 4}\left(-\frac{1}{2} \dot{u}^{2}+F(u)\right) d t
$$

where $\mathrm{T}$ is period of the nonlinear oscillator, $\partial F / \partial u=f$. Assume that its solution can be expressed as

$$
u(t)=A \cos (\omega t)
$$

where $A$ and $\omega$ are the amplitude and frequency of the oscillator, respectively. Substituting Eq. (3) into Eq. (2) results in:

$$
\begin{aligned}
J(A, \omega) & =\int_{0}^{T / 4}\left(-\frac{1}{2} A^{2} \omega^{2} \sin ^{2} \omega t+F(A \cos \omega t)\right) d t \\
& =\frac{1}{\omega} \int_{0}^{\pi / 2}\left(-\frac{1}{2} A^{2} \omega^{2} \sin ^{2} t+F(A \cos t)\right) d t \\
& =-\frac{1}{2} A^{2} \omega \int_{0}^{\pi / 2} \sin ^{2} t d t+\frac{1}{\omega} \int_{0}^{\pi / 2} F(A \cos t) d t
\end{aligned}
$$

Applying the Ritz method, it is required that

$$
\begin{aligned}
& \frac{\partial J}{\partial A}=0 \\
& \frac{\partial J}{\partial \omega}=0
\end{aligned}
$$

However with a careful inspection, for most cases we find that

$$
\frac{\partial J}{\partial \omega}=-\frac{1}{2} A^{2} \int_{0}^{\pi / 2} \sin ^{2} t d t-\frac{1}{\omega^{2}} \int_{0}^{\pi / 2} F(A \cos t) d t<0
$$

Thus, we modify the conditions in Eqs (4) and (7) into a simpler form:

$$
\frac{\partial J}{\partial \omega}=0
$$

from which the relationship between the amplitude and frequency of the oscillator can be obtained. 


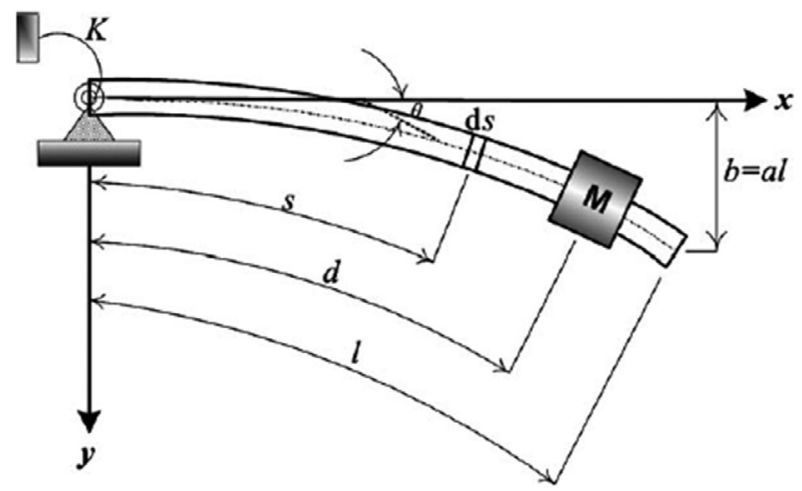

Fig. 1. Geometry and coordinate system for a beam with a lumped mass [31].

\section{Basic idea of Runge-Kutta's algorithm (RK)}

For a boundary value problem given, some numerical methods have been developed. Here, we apply the fourthorder RK algorithm to solve the governing equations subject to the given boundary conditions. The well-known and widely used RK iterative formulae for the second-order differential equations are:

$$
\begin{aligned}
& \dot{u}_{(i+1)}=\dot{u}_{i}+\frac{\Delta t}{6}\left(k_{1}+2 k_{2}+2 k_{3}+k_{4}\right), \\
& u_{(i+1)}=u_{i}+\Delta t\left[\dot{u}_{i}+\frac{\Delta t}{6}\left(k_{1}+k_{2}+k_{3}\right)\right],
\end{aligned}
$$

where $\Delta t$ is the increment of the time and $k_{1}, k_{2}, k_{3}$ and $k_{4}$ are determined from the following formulas:

$$
\begin{aligned}
& k_{1}=f\left(t_{i}, u_{i}, \dot{u}_{i}\right), \\
& k_{2}=f\left(t_{i}+\frac{\Delta t}{2}, u_{i}+\frac{\Delta t}{2}, \dot{u}_{i}+\frac{\Delta t}{2} k_{1}\right), \\
& k_{3}=f\left(t_{i}+\frac{\Delta t}{2}, u_{i}+\frac{\Delta t}{2} \dot{u}_{i}, \frac{1}{4} \Delta t^{2} k_{1}, \dot{u}_{i}+\frac{\Delta t}{2} k_{2}\right),
\end{aligned}
$$

The numerical solution starts from the boundary at the initial time, where the first value of the displacement function and its first-order derivative are determined from the initial conditions. Then, with a small time increment $[\Delta t]$, the displacement function and its first-order derivative at the new position can be obtained using Eq. (10). This process continues until the convergence criterion in satisfied.

\section{Applications of the variational approach}

In order to assess the advantages and the accuracy of the Variational Approach, let us consider the following examples:

\subsection{Example 1}

Hamdan [31] presented the Eq. (11) for a restrained uniform beam carrying an intermediate lumped mass. Figure 1 shows the schematic representation of the free vibration of a beam carrying a lumped mass along its span.

$$
\ddot{u}+\lambda u+\varepsilon_{1} u^{2} \ddot{u}+\varepsilon_{1} u \dot{u}^{2}+\varepsilon_{2} u^{4} \ddot{u}+2 \varepsilon_{2} u^{3} \dot{u}^{2}+\varepsilon_{3} u^{3}+\varepsilon_{4} u^{5}=0, \quad u(0)=A, \quad \dot{u}(0)=0
$$

The motion is assumed to start from the position of maximum displacement with zero initial velocity. $\lambda$ is an integer which may take values of 1,0 or -1 , and $\varepsilon_{1}, \varepsilon_{2}, \varepsilon_{3}$ and $\varepsilon_{4}$ are positive parameters [31]. 
Its variational formulation can be readily obtained from Eq. (2) as follows:

$$
J(u)=\int_{0}^{t}\left(\frac{1}{2} \dot{u}^{2}+\frac{1}{2} \lambda u^{2}+\frac{1}{2} \varepsilon_{1} \dot{u}^{2} u^{2}+\frac{1}{2} \varepsilon_{2} \dot{u}^{2} u^{4}+\frac{1}{4} \varepsilon_{3} u^{4}+\frac{1}{6} \varepsilon_{4} u^{6}\right) d t
$$

Choosing the trial function $u(t)=A \cos (\omega t)$ and substituting into Eq. (12), we obtain:

$$
J(A)=\int_{0}^{T / 4}\left(\begin{array}{l}
\frac{1}{2} A^{2} \omega^{2} \sin ^{2}(\omega t)+\frac{1}{2} \lambda A^{2} \cos ^{2}(\omega t)+\frac{1}{2} \varepsilon_{1} A^{4} \omega^{2} \sin ^{2}(\omega t) \cos ^{2}(\omega t) \\
+\frac{1}{2} \varepsilon_{2} A^{6} \omega^{2} \sin ^{2}(\omega t) \cos ^{4}(\omega t)+\frac{1}{4} \varepsilon_{3} A^{4} \cos ^{4}(\omega t)+\frac{1}{6} \varepsilon_{4} A^{6} \cos ^{6}(\omega t)
\end{array}\right) d t
$$

The stationary condition with respect to $A$ leads to:

$$
\frac{\partial J}{\partial A}=\int_{0}^{T / 4}\left(\begin{array}{l}
A \omega^{2} \sin ^{2}(\omega t)+\lambda A \cos ^{2}(\omega t)+2 \varepsilon_{1} A^{3} \omega^{2} \sin ^{2}(\omega t) \cos ^{2}(\omega t) \\
+3 \varepsilon_{2} A^{5} \omega^{2} \sin ^{2}(\omega t) \cos ^{4}(\omega t)+\varepsilon_{3} A^{3} \cos ^{4}(\omega t)+\varepsilon_{4} A^{5} \cos ^{6}(\omega t)
\end{array}\right) d t=0
$$

Or in a more simplified form:

$$
\frac{\partial J}{\partial A}=\int_{0}^{\pi / 2}\left(\begin{array}{l}
A \omega^{2} \sin ^{2} t+\lambda A \cos ^{2} t+2 \varepsilon_{1} A^{3} \omega^{2} \sin ^{2} t \cos ^{2} t \\
+3 \varepsilon_{2} A^{5} \omega^{2} \sin ^{2} t \cos ^{4} t+\varepsilon_{3} A^{3} \cos ^{4} t+\varepsilon_{4} A^{5} \cos ^{6} t
\end{array}\right) d t=0
$$

Solving Eq. (15) for $\omega$, we have:

$$
\omega^{2}=\frac{\int_{0}^{\frac{\pi}{2}}\left(\lambda A \cos ^{2} t+\varepsilon_{3} A^{3} \cos ^{4} t+\varepsilon_{4} A^{5} \cos ^{6} t\right) d t}{\int_{0}^{\frac{\pi}{2}}\left(A \sin ^{2} t+2 \varepsilon_{1} A^{3} \sin ^{2} t \cos ^{2} t+3 \varepsilon_{2} A^{5} \sin ^{2} t \cos ^{4} t\right) d t}
$$

Thus,

$$
\omega_{V A}=\sqrt{\frac{5 \varepsilon_{4} A^{4}+6 \varepsilon_{3} A^{2}+8 \lambda}{3 A^{4} \varepsilon_{2}+4 A^{2} \varepsilon_{1}+8}}
$$

According to Eqs (3) and (17), we can obtain the following approximate solution:

$$
u(t)=A \cos \left(\sqrt{\frac{5 \varepsilon_{4} A^{4}+6 \varepsilon_{3} A^{2}+8 \lambda}{3 A^{4} \varepsilon_{2}+4 A^{2} \varepsilon_{1}+8}} t\right)
$$

\subsection{Example 2}

In this example, we have considered the Duffing equation with constant coefficients as shown in Fig. 2. It may be easily verified that the governing equation of the oscillation is as follow:

$$
\ddot{x}+\frac{k_{1}}{m} x+\frac{k_{2}}{2 m h^{2}} x^{3}=\frac{F_{0}}{m} \sin \omega_{0} t, \quad x(0)=A, \quad \dot{x}(0)=0
$$

where $x$ is the dimensionless displacement and $t$ is the time variable.

Its variational formulation can be readily obtained from Eq. (2) as follows:

$$
J(x)=\int_{0}^{t}\left(\frac{1}{2} \dot{x}^{2}+\frac{1}{2} \frac{k_{1}}{m} x^{2}+\frac{1}{8} \frac{k_{2}}{m h^{2}} x^{4}-\frac{F_{0} \sin \left(\omega_{0} t\right)}{m} x\right) d t
$$

Choosing the trial function $x(t)=A \cos (\omega t)$ and substituting into Eq. (20), we obtain:

$$
\begin{aligned}
J(A) & =\int_{0}^{T / 4}\left(\frac{1}{2} A^{2} \omega^{2} \sin ^{2}(\omega t)+\frac{1}{2} \frac{k_{1}}{m} A^{2} \cos ^{2}(\omega t)+\frac{1}{8} \frac{k_{2}}{m h^{2}} A^{4} \cos ^{4}(\omega t)\right. \\
& \left.-\frac{F_{0} \sin \left(\omega_{0} t\right)}{m} A \cos (\omega t)\right) d t
\end{aligned}
$$




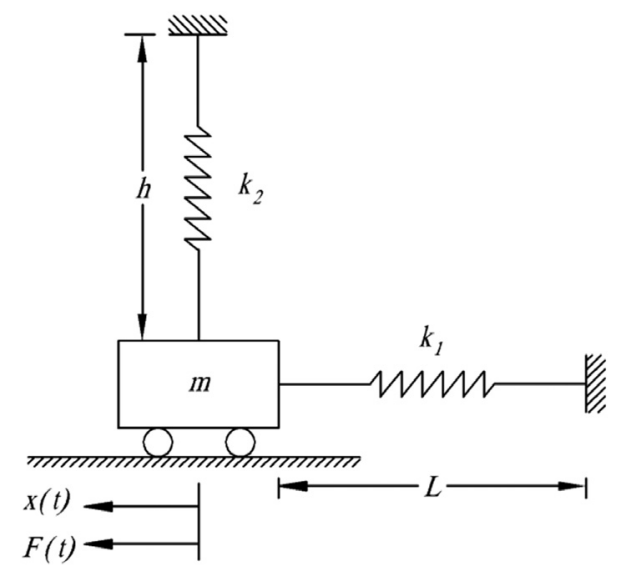

Fig. 2. The physical model of Duffing equation with constant coefficients.

The stationary condition with respect to $A$ leads to:

$$
\frac{\partial J}{\partial A}=\int_{0}^{T / 4}\left(A \omega^{2} \sin ^{2}(\omega t)+A \frac{k_{1}}{m} \cos ^{2}(\omega t)+\frac{1}{2} \frac{k_{2}}{m h^{2}} A^{3} \cos ^{4}(\omega t)-\frac{F_{0} \sin \left(\omega_{0} t\right)}{m} \cos (\omega t)\right) d t=0
$$

Or in more simplified form:

$$
\frac{\partial J}{\partial A}=\int_{0}^{\pi / 2}\left(A \omega^{2} \sin ^{2} t+A \frac{k_{1}}{m} \cos ^{2} t+\frac{1}{2} \frac{k_{2}}{m h^{2}} A^{3} \cos ^{4} t-\frac{F_{0} \sin \left(\omega_{0} t\right)}{m} \cos t\right) d t=0
$$

Solving Eq. (23) for $\omega$, we have:

$$
\omega^{2}=\frac{\int_{0}^{\frac{\pi}{2}}\left(A \frac{k_{1}}{m} \cos ^{2} t+\frac{1}{2} \frac{k_{2}}{m h^{2}} A^{3} \cos ^{4} t-\frac{F_{0} \sin \left(\omega_{0} t\right)}{m} \cos t\right) d t}{\int_{0}^{\frac{\pi}{2}}\left(A \sin ^{2} t\right) d t}
$$

Thus,

$$
\omega_{V A}=\frac{1}{4} \sqrt{2} \sqrt{\frac{8 k_{1} A h^{2} \pi-32 F_{0} \sin \left(\omega_{0} t\right) h^{2}+3 k_{2} A^{3} \pi}{m h^{2} \pi A}}
$$

According to Eqs (3) and (25), we can obtain the following approximate solution:

$$
x(t)=A \cos \left(\frac{1}{4} \sqrt{2} \sqrt{\frac{8 k_{1} A h^{2} \pi-32 F_{0} \sin \left(\omega_{0} t\right) h^{2}+3 k_{2} A^{3} \pi}{m h^{2} \pi A}} t\right)
$$

\section{Results and discussions}

To illustrate and verify the accuracy of this new approximate analytical approach, some comparisons of the time history oscillatory displacement responses with those of the numerical solution using Runge-Kutta algorithm are presented for Example 1 in Table 1 and Figs 3-6, and for Example 2 in Table 2 and Figs 7-10. The values of parameters $\varepsilon_{1}, \varepsilon_{2}, \varepsilon_{3}, \varepsilon_{4}$ associated with each of the six calculation modes are shown in Table 1.

Table 1 gives the comparison of the obtained results with those obtained by Qian et al. [36] and Runge-Kutta algorithm for different values of $\lambda, \varepsilon_{1}, \varepsilon_{2}, \varepsilon_{3}, \varepsilon_{4}$ and different initial conditions. It can be observed from Table 1 that there is high level of agreement between the results obtained from the variational approach and those by Qian et al. [36] and Runge-Kutta algorithm. The maximum relative error between the variational approach results and the numerical results is $0.068 \%$. Figure 3 represents a comparison of the analytical solution of $u(t)$ based on time with the numerical solution. The time history diagram of $u(t)$ starts without an apparent deviation with $A=0.3$. The 
Table 1

The comparison of the numerical and analytically derived frequencies corresponding to various parameters in Eq. (17)

\begin{tabular}{|c|c|c|c|c|c|c|c|c|c|}
\hline \multicolumn{6}{|c|}{ Constant parameter } & \multicolumn{2}{|c|}{ Approximate solution } & \multirow{2}{*}{$\begin{array}{c}\text { Numerical solution } \\
\omega_{N u m} \\
\end{array}$} & \multirow{2}{*}{$\begin{array}{l}\text { Relative error }(\%) \\
\frac{\left(\omega_{V A}-\omega_{N u m}\right)}{\omega_{N M}}\end{array}$} \\
\hline$A$ & $\lambda$ & $\varepsilon_{1}$ & $\varepsilon_{2}$ & $\varepsilon_{3}$ & $\varepsilon_{4}$ & $\omega_{V A}$ & $\omega_{H A M}[36]$ & & \\
\hline 0.2 & 1 & 4.0515 & 1.6652 & 0.2814 & 0.1497 & 0.9655 & 0.9665 & 0.9666 & 0.1211 \\
\hline 0.3 & 1 & 8.2056 & 3.1454 & 0.2723 & 0.1337 & 0.8597 & 0.8714 & 0.8643 & 0.5274 \\
\hline 0.5 & 1 & 1.6420 & 0.9131 & 0.3136 & 0.2043 & 0.9326 & 0.9386 & 0.9364 & 0.4094 \\
\hline 0.8 & 1 & 0.8940 & 0.4674 & 0.3136 & 0.2043 & 0.9412 & - & 0.9477 & 0.6865 \\
\hline 1 & 1 & 0.3268 & 0.1296 & 0.2326 & 0.0876 & 1.0071 & 1.0123 & 1.0102 & 0.3059 \\
\hline 1.2 & 1 & 0.3038 & 0.1151 & 0.2326 & 0.0876 & 1.0214 & - & 1.0283 & 0.6795 \\
\hline
\end{tabular}

Table 2

The comparison of the variational approach solution and those of the energy balance method and Runge-Kutta algorithm

\begin{tabular}{|c|c|c|c|c|c|c|c|}
\hline \multicolumn{4}{|c|}{ Case 1} & \multicolumn{4}{|c|}{ Case 2} \\
\hline$t$ & $X(t)_{V A}$ & $X(t)_{E B M}[35]$ & $X(t)_{R K}$ & $t$ & $X(t)_{V A}$ & $X(t)_{E B M}[35]$ & $X(t)_{R K}$ \\
\hline 0 & 0.5 & 0.5 & 0.5 & 0 & 0.2 & 0.2 & 0.2 \\
\hline 0.4 & -0.3290 & -0.3292 & -0.3269 & 0.4 & -0.1948 & -0.1948 & -0.1927 \\
\hline 0.8 & -0.0695 & -0.0688 & -0.0563 & 0.8 & 0.1842 & 0.1841 & 0.1798 \\
\hline 1.2 & 0.4169 & 0.4165 & 0.4119 & 1.2 & -0.1622 & -0.1621 & -0.1615 \\
\hline 1.6 & -0.4855 & -0.4855 & -0.4851 & 1.6 & 0.1209 & 0.1218 & 0.1349 \\
\hline 2 & 0.2462 & 0.2450 & 0.2265 & 2 & -0.1050 & -0.1043 & -0.0991 \\
\hline 2.4 & 0.1535 & 0.1555 & 0.1734 & 2.4 & 0.0682 & 0.0674 & 0.0571 \\
\hline 2.8 & -0.4608 & -0.4614 & -0.4694 & 2.8 & 0.0084 & 0.0064 & -0.0143 \\
\hline 3.2 & 0.4400 & 0.4402 & 0.4407 & 3.2 & -0.0188 & -0.0194 & -0.0258 \\
\hline 3.6 & -0.0868 & -0.0893 & -0.1132 & 3.6 & 0.0456 & 0.0474 & 0.0639 \\
\hline 4 & -0.3206 & -0.3179 & -0.2806 & 4 & -0.1318 & -0.1299 & -0.1018 \\
\hline 4.4 & 0.4991 & 0.4990 & 0.4986 & 4.4 & 0.1395 & 0.1395 & 0.1383 \\
\hline 4.8 & -0.3788 & -0.3783 & -0.3700 & 4.8 & -0.1432 & -0.1453 & -0.1686 \\
\hline
\end{tabular}

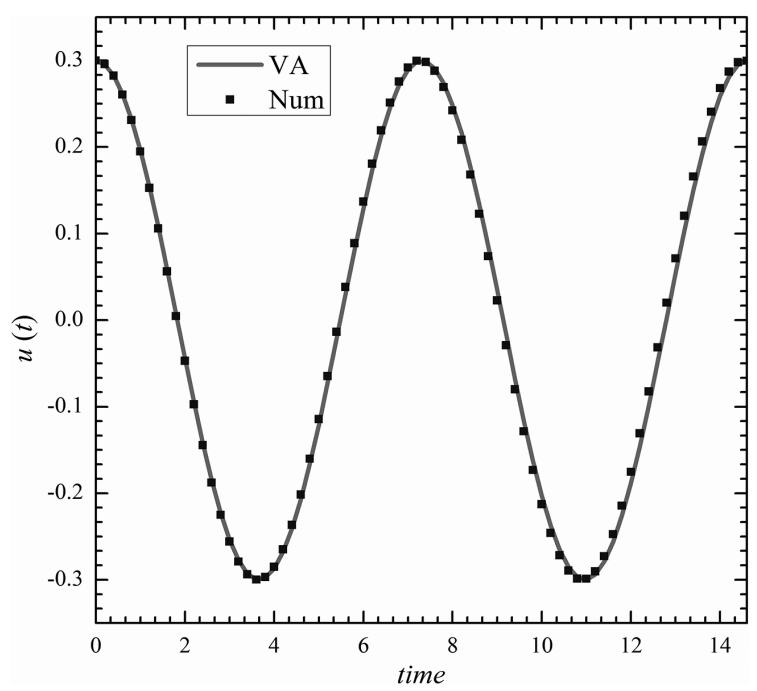

Fig. 3. The comparison of the analytical solution of $u(t)$ with the numerical solution for $\varepsilon_{1}=8.20557, \varepsilon_{2}=3.145368, \varepsilon_{3}=0.272313$, $\varepsilon_{4}=0.133708, \lambda=1, A=0.3$.

motion of the system is a periodic motion and the amplitude of the vibration is a function of the initial conditions. The best accuracy can be seen at the extreme points. Although the deviation of the solutions is expected to increase as time progresses, the analytical solutions have adequate accuracy for the period shown. Figures 4(a)-4(d) show the phase plan curves $\left(\dot{u}(t)\right.$ versus $u(t)$ curve) of Eq. (18) to show the effect of the small parameters $\varepsilon_{1}, \varepsilon_{2}, \varepsilon_{3}, \varepsilon_{4}$. The phase plots show the behavior of the oscillator when the constant parameters are changed. It is periodic with its center at $(0,0)$. This situation also occurs in the unforced, undamped cubic Duffing oscillators. 


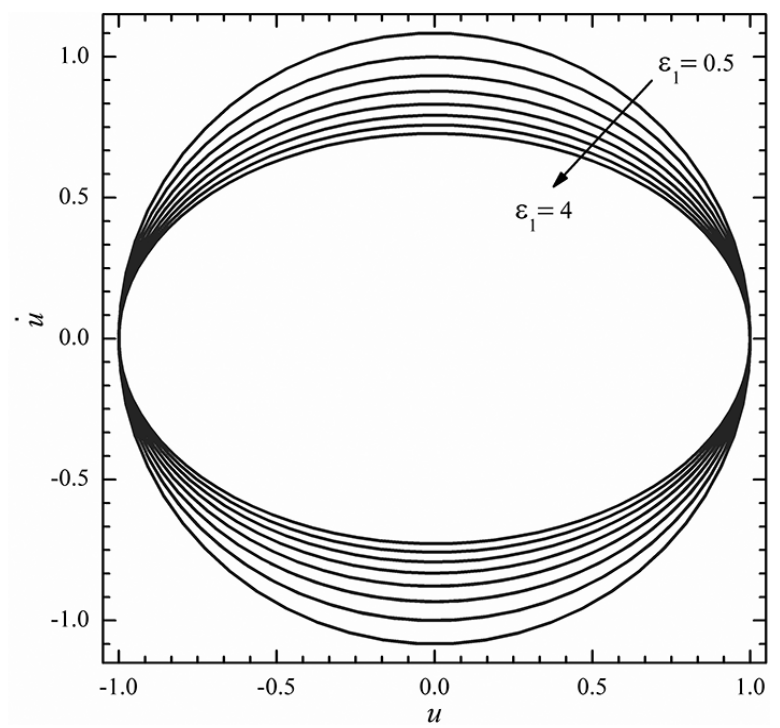

(a) The phase plan for $A=1, \lambda=1, \varepsilon_{2}=0.5, \varepsilon_{3}=0.5, \varepsilon_{4}=0.5$

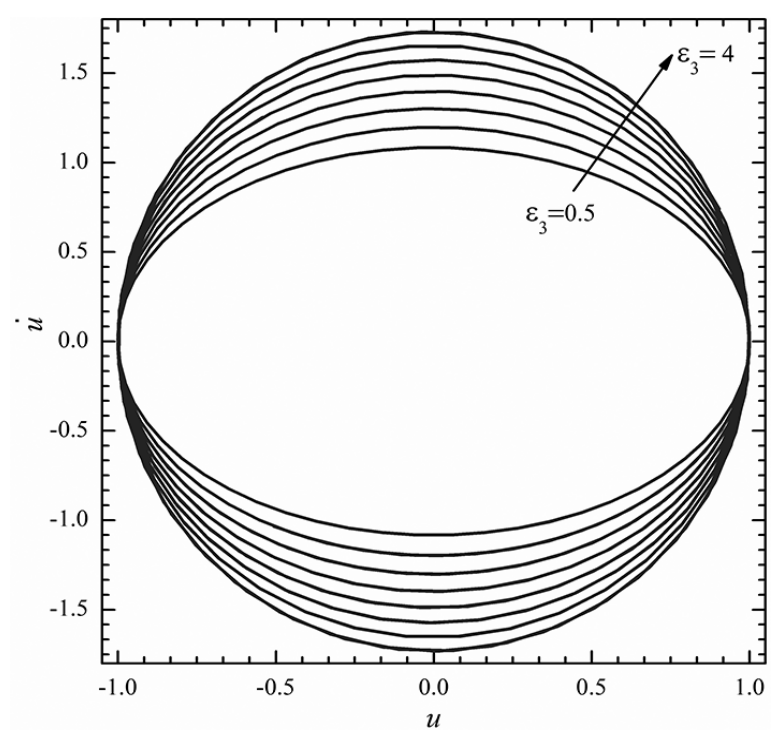

(c) The phase plan for $A=1, \lambda=1, \varepsilon_{1}=0.5, \varepsilon_{2}=0.5, \varepsilon_{4}=0.5$

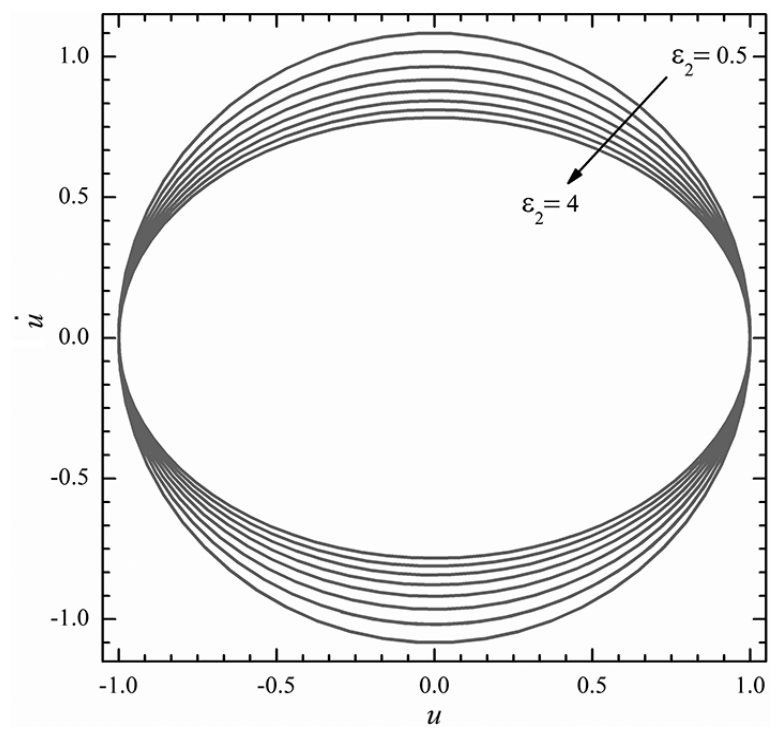

(b) The phase plan for $A=1, \lambda=1, \varepsilon_{1}=0.5, \varepsilon_{3}=0.5, \varepsilon_{4}=0.5$

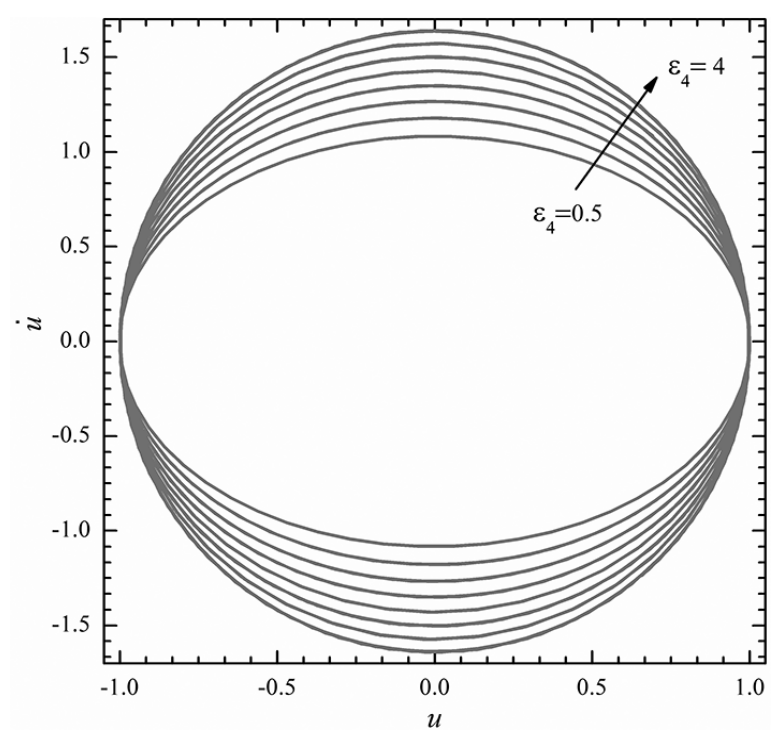

(d) The phase plan for $A=1, \lambda=1, \varepsilon_{1}=0.5, \varepsilon_{2}=0.5, \varepsilon_{3}=0.5$

Fig. 4. a. The phase plan for $A=1, \lambda=1, \varepsilon_{2}=0.5, \varepsilon_{3}=0.5, \varepsilon_{4}=0.5 \mathrm{~b}$. The phase plan for $A=1, \lambda=1, \varepsilon_{1}=0.5, \varepsilon_{3}=0.5, \varepsilon_{4}=0.5$ c. The phase plan for $A=1, \lambda=1, \varepsilon_{1}=0.5, \varepsilon_{2}=0.5, \varepsilon_{4}=0.5 \mathrm{~d}$. The phase plan for $A=1, \lambda=1, \varepsilon_{1}=0.5, \varepsilon_{2}=0.5, \varepsilon_{3}=0.5$.

The variation of frequency with respect to amplitude $(A)$ and $\varepsilon_{1}$ and $\varepsilon_{4}$ at $\lambda=1, \varepsilon_{2}=0.8, \varepsilon_{3}=0.2, \varepsilon_{4}=2$ are shown in Figs 5 and 6. These figures show that the amplitude of the vibration $(A)$ has a greater effect on the frequency than the constant parameters $\varepsilon_{1}, \varepsilon_{2}, \varepsilon_{3}, \varepsilon_{4}$.

For the second example, Table 2 presents the comparison of VA with Rung-kutta algorithm and the energy balance method for the following two cases:

Case 1 where, $A=0.5, L=1 \mathrm{~m}, h=0.8 \mathrm{~m}, m=10 \mathrm{~kg}, k_{1}=300 \mathrm{~N} / \mathrm{m}, k_{2}=200 \mathrm{~N} / \mathrm{m}, F_{0}=1 \mathrm{~N}$, $\omega_{0}=2 \mathrm{rad} / \mathrm{s}$.

Case 2 where, $A=0.2, L=1 \mathrm{~m}, h=0.5 \mathrm{~m}, m=10 \mathrm{~kg}, k_{1}=500 \mathrm{~N} / \mathrm{m}, k_{2}=600 \mathrm{~N} / \mathrm{m}, F_{0}=1 \mathrm{~N}$, $\omega_{0}=5 \mathrm{rad} / \mathrm{s}$. 


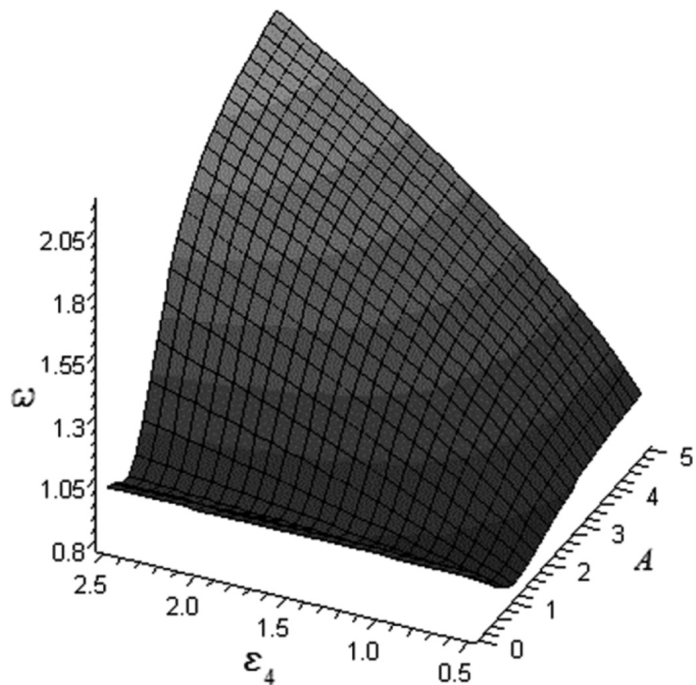

Fig. 5. Sensitivity analysis of frequency for $0<A<5, \lambda=1$, $0.5<\varepsilon_{1}<2.5, \varepsilon_{2}=0.8, \varepsilon_{3}=0.2, \varepsilon_{4}=2$.

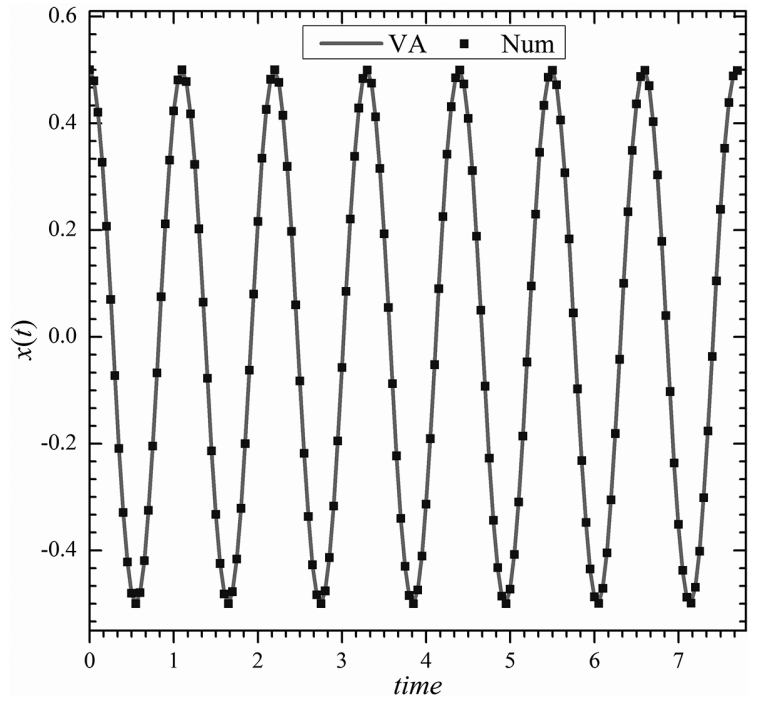

Fig. 7. The comparison of the analytical solution of $x(t)$ with the numerical solution for $A=0.5$.

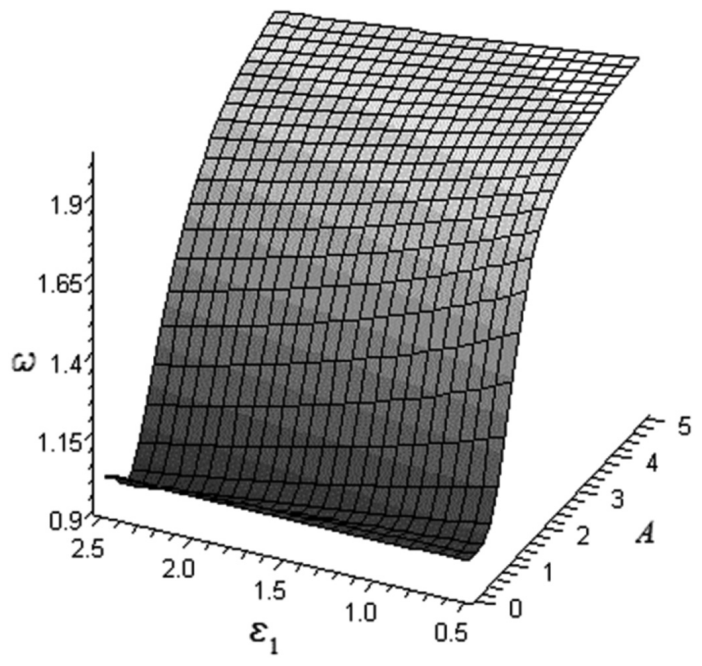

Fig. 6. Sensitivity analysis of frequency for $0<A<5, \lambda=1$, $\varepsilon_{1}=2, \varepsilon_{2}=0.8, \varepsilon_{3}=0.2,0.5<\varepsilon_{4}<2.5$.

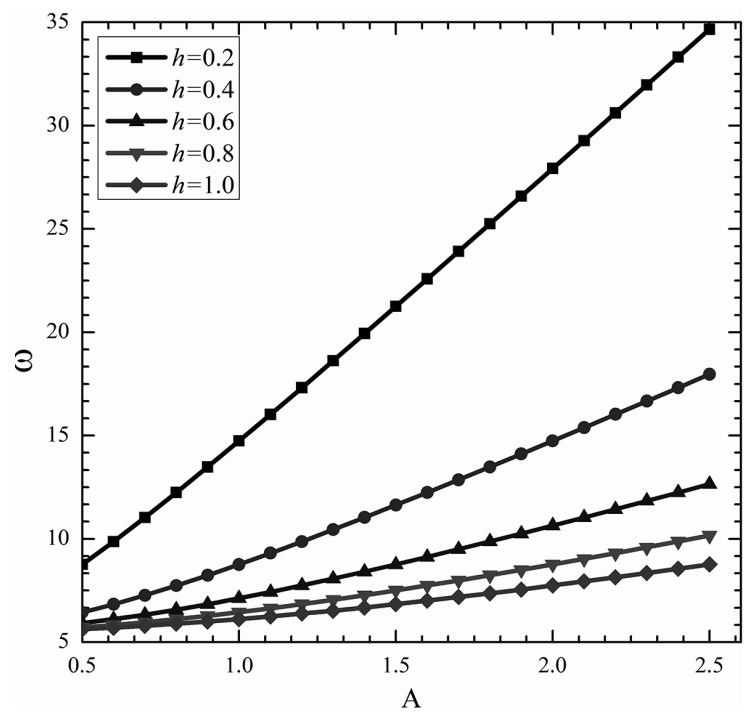

Fig. 8. The comparison of the frequency corresponding to various parameters of amplitude $(A)$ for $m=10 \mathrm{~kg}, k_{1}=300, k_{2}=200$, $F_{0}=1 \mathrm{~N}, \omega_{0}=2$.

The VA solutions demonstrate excellent agreement with those of Runge-Kutta algorithm and energy balance method. Figure 7 shows that the behavior of the system is periodic and that it is a function of the initial conditions. For a better understanding of the motion of the considered system, the effect of $h$ is shown in Fig. 8. The influence of $h$ becomes more apparent when $h$ has smaller values. The comparison of the frequency corresponding to various parameters such as the amplitude $(A)$ and the stiffness of springs $k_{1}, k_{2}$ for $m=10, h=1, F_{0}=1, \omega_{0}=2$ have been studied in Figs 9 and 10. These figures represent the significant effects of $k_{1}, k_{2}$ on the nonlinear frequency of the system for different values of amplitude. It is evident that VA shows an excellent agreement with the numerical solutions VA is quickly convergent and is valid for a wide range of vibration amplitudes and initial conditions. The accuracy of the results shows that the VA can be potentially used for the analysis of strongly nonlinear oscillation problems accurately. 


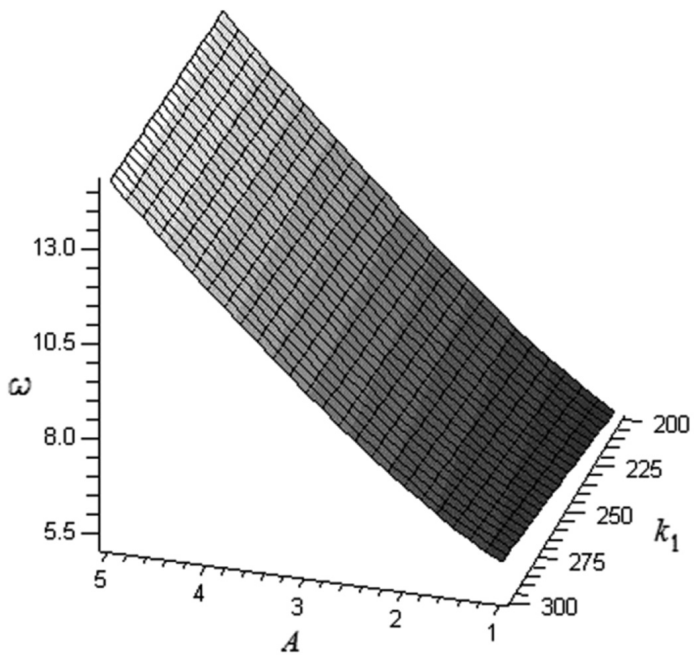

Fig. 9. Sensitivity analysis of frequency for $1<A<5, m=10$, $h=1,200<k_{1}<300, k_{2}=200, F_{0}=1, \omega_{0}=2$.

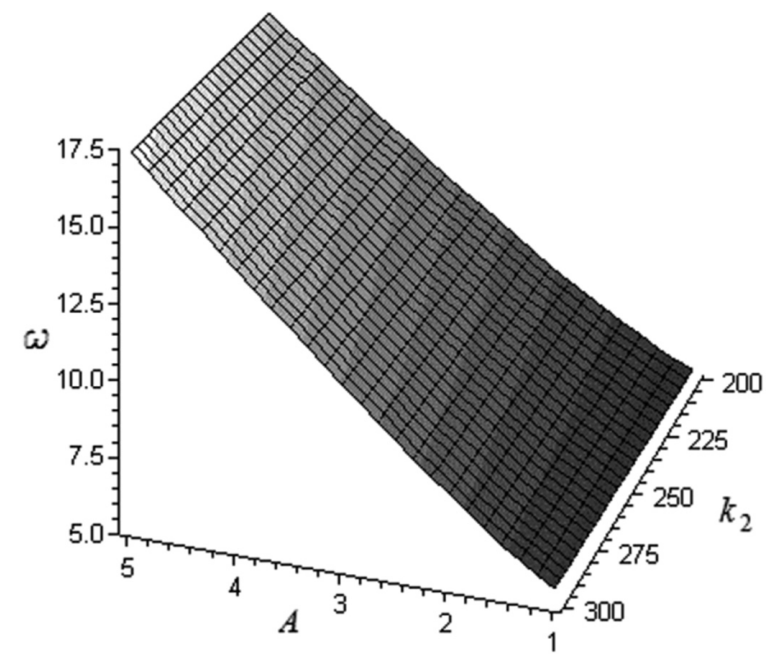

Fig. 10. Sensitivity analysis of frequency for $1<A<5, m=10$, $h=1, k_{1}=200,200<k_{2}<300, F_{0}=1, \omega_{0}=2$.

\section{Conclusions}

In this paper a quite uncomplicated but productive new method for non-natural oscillators called the Variational Approach has been used to obtain analytical solutions for the Duffing equation with cubic-quintic nonlinearities, which are not studied as extensively as other cubic nonlinearities. Comparing with other numerical results in the literature, it has been shown that the results of VA require smaller computational effort and only a first-order approximation, which leads to high accuracy solutions. The analytical solutions yield to a broader insight into the effects of various system parameters and initial conditions. Also, the analytical solutions give a reference frame for the verification and validation of other numerical approaches. Variational Approach can be simply extended as a powerful approximate analytical technique, which provides an effective and convenient mathematical tool for solving non-linear differential equations with quadratic and cubic nonlinearities.

\section{References}

[1] V.I. Arnold, Mathematical methods of classical mechanics, New York, Springer, 1978.

[2] M. Gitterman, The noisy oscillator: The first hundred years, from Einstein until now, World Scientific Publishing, Singapore, 2005.

[3] J. Guckenheimer and P. Holmes, Nonlinear oscillations, dynamical systems and bifurcations of vector fields, Springer-Verlag, New York, 1983.

[4] D.W. Jordan and P. Smith, Nonlinear ordinary differential equations: An introduction for scientists and engineers, Oxford University Press, New York, 2007.

[5] A.D. Polyanin and V.F. Zaitsev, Handbook of exact solutions for ordinary differential equations, 2nd edition, London, CRC Press, 2003.

[6] A.D. Polyanin, V.F. Zaitsev and A. Mousssiaux, Handbook of first order partial differential equations, London, Taylor \& Francis, 2002.

[7] M. Bayat, M. Shahidi, A. Barari and G. Domairry, The approximate analysis of nonlinear behavior of structure under harmonic loading, Int J Phy Sci 5(7) (2010), 1074-1080.

[8] H. Tari, D.D. Ganji and M. Rostamian, Approximate solutions of K(2,2), KdV and modified KdV equations by VIM, HPM and HAM, International Journal Of Nonlinear Sciences And Numerical Simulation 8(2) (2007), 203-210.

[9] M. Bayat, I. Pakar and M. Bayat, Application of He's Energy Balance Method for Pendulum attached to rolling wheels that are restrained by a spring, Int J Phy Sci 7(6) (2012), 913-921.

[10] M. Bayat, A. Barari and M.Shahidi, Dynamic response of axially loaded euler-bernoulli beams, Mechanika $17(2)$ (2011), $172-177$.

[11] I. Pakar and M. Bayat, Analytical solution for strongly nonlinear oscillation systems using Energy Balance Method, Int J Phy Sci 6(22) (2011), 5166-5170.

[12] M. Bayat and I. Pakar, Application of He's Energy Balance Method for nonlinear vibration of thin circular sector cylinder, Int J Phy Sci 6(23) (2011), 5564-5570.

[13] D.D. Ganji, M. Nourollahi and M. Rostamian, A comparison of variational iteration method with adomian's decomposition method in some highly nonlinear equations, International Journal of Science \& Technology 2(2) (2007), 179-188. 
[14] I. Pakar, M. Bayat and M. Bayat, On the approximate analytical solution for parametrically excited nonlinear oscillators, Journal of Vibroengineering 14(1) (2012), 423-429.

[15] M. Bayat, M. Bayat and M. Bayat, An analytical approach on a mass grounded by linear and nonlinear springs in series, Int J Phy Sci 6(2) (2011), 229-236.

[16] J.H. He, Variational approach for nonlinear oscillators, Chaos, Solitons and Fractals 34(5) (2007), 1430-1439.

[17] M. Shahidi, M. Bayat, I. Pakar and G.R. Abdollahzadeh, On the solution of free non-linear vibration of beams, Int J Phy Sci 6(7) (2011), 1628-1634.

[18] I. Pakar, M. Bayat and M. Bayat, Analytical evaluation of the nonlinear vibration of a solid circular sector object, Int J Phy Sci 6(30) (2011), 6861-6866.

[19] M. Bayat, M. Shahidi and M. Bayat, Application of iteration perturbation method for nonlinear oscillators with discontinuities, Int J Phy Sci 6(15) (2011), 3608-3612.

[20] M. Bayat and I. Pakar, Nonlinear free vibration analysis of tapered beams by hamiltonian approach, Journal of Vibroengineering 13(4) (2011), 654-661.

[21] D.Q. Zeng and Y.Y. Lee, Analysis of strongly nonlinear oscillator using the max-min approach, Int J Nonlinear Sci Numer Simul 10(10) (2009), 1361-1368.

[22] M. Bayat, I. Pakar and M. Bayat, Analytical study on the vibration frequencies of tapered beams, Latin American Journal of Solids and Structures 8(2) (2011), 149-162.

[23] Y.Y. Shen and L.F. Mo, The max-min approach to a relativistic equation, Comput Math Appl 58(11-12) (2009), 2131-2133.

[24] M. Bayat, I. Pakar and M. Shahidi, Analysis of nonlinear vibration of coupled systems with cubic nonlinearity, Mechanika 17(6) (2011), 620-629.

[25] A. Kimiaeifar, A.R. Saidi, A.R. Sohouli and D.D. Ganji, Analysis of modified van der pol's oscillator using he's parameter-expanding methods, Current Applied Physics 10(1) (2010), 79-283.

[26] J.H. He, An improved amplitude-frequency formulation for nonlinear oscillators, International Journal of Nonlinear Sciences and Numerical Simulation 9(2) (2008), 211-212.

[27] M. Bayat and G.R. Abdollahzadeh, On the effect of the near field records on the steel braced frames equipped with energy dissipating devices, Latin American Journal of Solids and Structures 8(4) (2011), 429-443.

[28] E. Ghasemi, M. Bayat and M. Bayat, Visco-elastic MHD flow of walters liquid b fluid and heat transfer over a non-isothermal stretching sheet, Int J Phy Sci 6(21) (2011), 5022-5039.

[29] I. Pakar and M. Bayat, Analytical study on the non-linear vibration of Euler-Bernoulli beams, Journal of Vibroengineering 14(1) (2012), 216-224.

[30] M.H. Mohammadi, A. Mohammadi, A. Kimiaeifar and H. Tabaei, Application of HPEM to investigate the response and stability of nonlinear problems in vibration, Australian Journal of Basic and Applied science 4(4) (2010), 557-563.

[31] M.N. Hamden and N.H. Shabaneh, On the large amplitude free vibrations of a restrained uniform beam carrying an intermediate lumped mass, Journal of Sound and Vibration 199(5) (1997), 711-736.

[32] Q.Q. Hu, C.W. Lim and L.Q. Chen, Nonlinear vibration of a cantilever with a Derjaguin-Müller-Toporov contact end, Int J Struct Stab Dyn 8(1) (2008), 25-40.

[33] L.L. Ke, J. Yang, S. Kitipornchai and Y. Xiang, Flexural vibration and elastic buckling of a cracked Timoshenko beam made of functionally graded materials, Mech Adv Mater Struct 16(6) (2009), 488-502.

[34] S.S. Chen and C.K. Chen, Application of the differential transformation method to the free vibrations of strongly non-linear oscillators, Nonlinear Anal Real World Appl 10(2) (2009), 881-888.

[35] I. Mehdipour, D.D. Ganji and M. Mozaffari, Application of the energy balance method to nonlinear vibrating equations, Current Applied Physics 10(1) (2010), 104-112.

[36] Y.H. Qian, S.K. Lai, W. Zhang and Y. Xiang, Study on asymptotic analytical solutions using HAM for strongly nonlinear vibrations of a restrained cantilever beam with an intermediate lumped mass, Numer Algor 58(3) (2011), 293-314. 

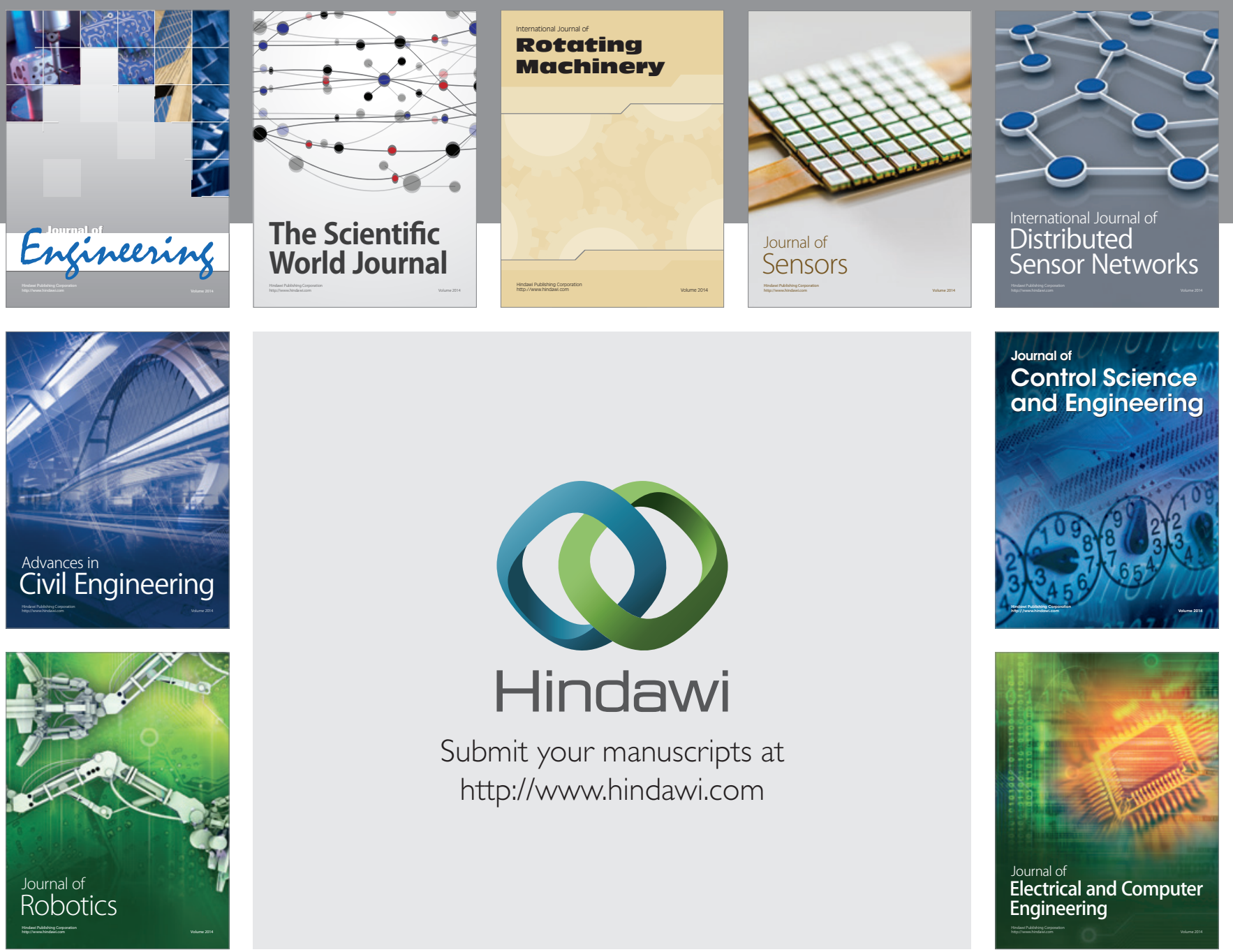

Submit your manuscripts at

http://www.hindawi.com
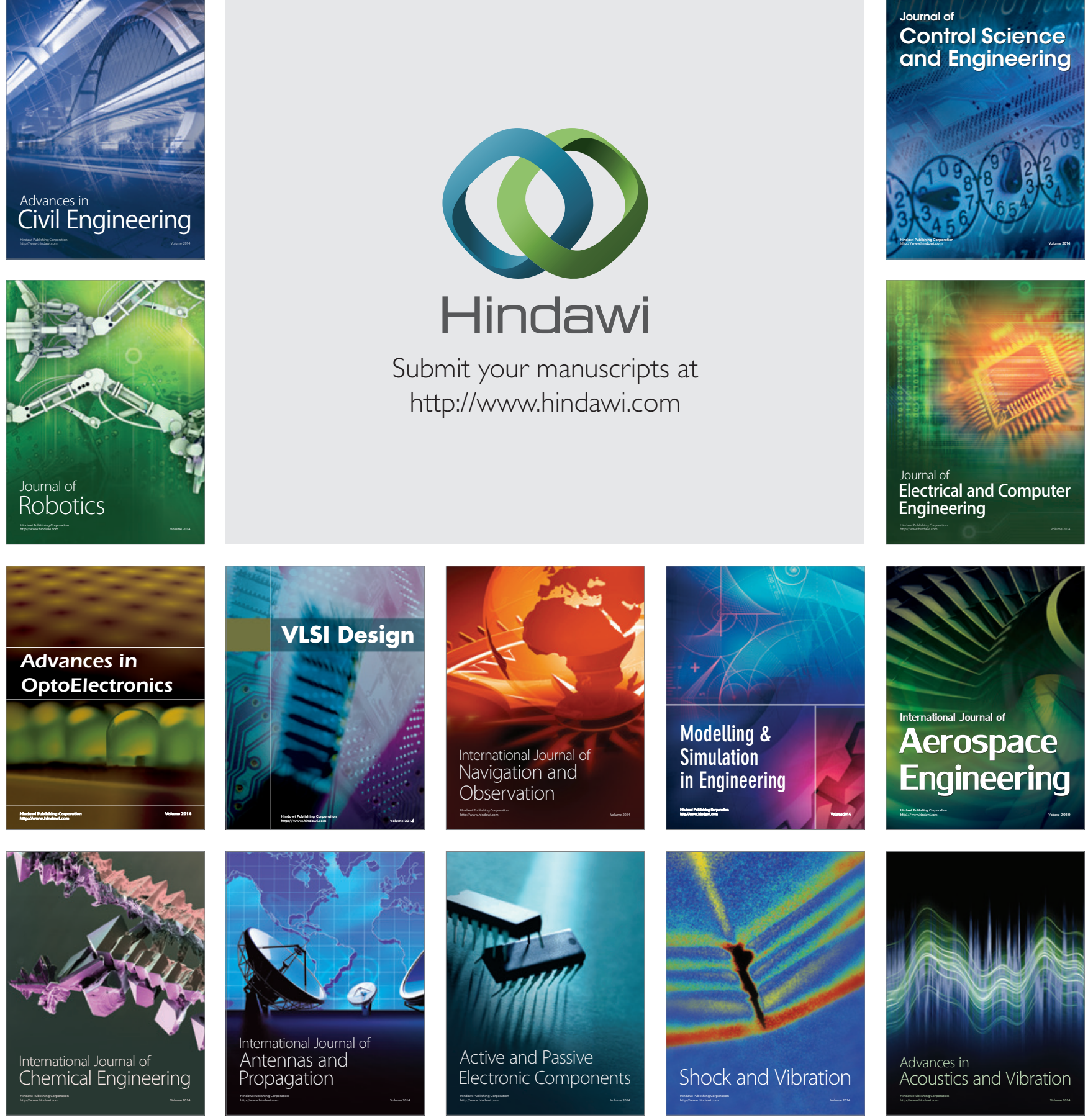